TOKYO J. MATH.

VOL. 31, No. 2, 2008

\title{
Global Classical Solutions of IBVP to Nonlinear Equation of a Suspended String
}

\author{
Jaipong WONGSAWASDI and Masaru YAMAGUCHI
}

Tokai University

(Communicated by M. Tanaka)

\begin{abstract}
We are concerned with the existence and uniqueness of the classical solution to IBVP for a nonlinear equation of a suspended string with uniform density to which a monotonous nonlinear time-independent outer force works. For this purpose we derive the higher order energy estimates, and employ the Galerkin method combining with the compactness argument along the refined method of [Sath]. We need the regularity theory of Nirenberg type for the suspended string operator $L$.
\end{abstract}

\section{Introduction}

Let $\Omega$ be a cylindrical domain $(0, a) \times(0, T)$. Consider IBVP for a nonlinear equation of a flexible and heavy suspended string of finite length $a$ with a uniform density

(P)

$$
\begin{cases}\partial_{t}^{2} u(x, t)+L u(x, t)+u(x, t)^{3}=0, & (x, t) \in \Omega, \\ u(a, t)=0, \quad t \in(0, T), & \\ u(x, 0)=\phi(x), \quad \partial_{t} u(x, 0)=\psi(x), & x \in(0, a),\end{cases}
$$

where $L$ is a second order differential operator of the form

$$
L=L_{0}\left(x, \partial_{x}\right)=-\left(x \partial_{x}^{2}+\partial_{x}\right)
$$

The operator $L$ is a particular form of suspended string equations introduced by [K-G-S] and [Ko]. As for IBVP to the linear equation of the suspended string with the quasi-periodic forcing term, the existence of almost periodic $C^{2}$-solutions was proved in [Ya1], and for several periodic problems of nonlinear equation of suspended string, see [Ya2] and [Ya-Na-Ma]. Quite recently, the present authors proved the existence of time-global weak solutions to these equations with the blowing-up term like $|u|^{p-1} u, p>1$, by using the potential well method (see [Wo-Ya]).

Received February 7, 2007; revised February 23, 2007

This research is supported in part by Grant-in-Aid for Scientific Research (C)(2) No. 15540213, Japan Society for the Promotion of Science. 
The purpose of this paper is to show the existence and uniqueness of a classical solution of $(\mathrm{P})$ with a monotonous cubic nonlinear term $u^{3}$. It will be shown that the unique classical solution exists for any large initial data (see Main Theorem in §2), while in [Wo-Ya] some boundedness condition related to the potential well on the initial data is assumed. To prove our result we employ the Galerkin method combining with the higher order energy estimates of approximate solutions and compactness argument in the refined way of [Sath], which proved the existence and uniqueness of the classical solution to a nonlinear 3D wave equation. Contrary to [Sath], the difficulty of our problem comes from the singularity of solutions at $x=0$. We note that $L$ is the special case of the differential operator $L_{\mu}$ (see $\S 3$ ), and degenerates at the origin. Fortunately, the second author made some appropriate function spaces suitable for suspended string operators $L_{\mu}$ (see [Ya1], [Ya2]). As will be seen, our argument will be quite effective in Lebesgue and Sobolev type spaces with some power weight of $x$. In $\S 2$ we review the definitions of these function spaces, and in $\S 3$ we will provide the properties of the operator $L$ on these spaces and some Poincaré-Sobolev type inequalities suited for these spaces (see [Ya1], [Ya2], [Ya-Na-Ma], [Wo-Ya]). It will be seen that these spaces have an algebraic property for an appropriate regularity (see Lemma 4.3), which plays important roles in our argument. In $\S 4$ we derive the higher order energy estimates of the approximate solutions, and $\$ 5$ will be devoted to prove our main theorem. The strategy is to use these higher order energy estimates and the compactness method in the standard way. We need the elliptic regularity theory of Nirenberg type for our operator $L$ which enables us to obtain the appropriate regularity of solutions. In the last section this elliptic regularity theorem will be proved as an appendix.

\section{Function spaces and Main Theorem}

Let $R_{+}^{1}$ and $Z_{+}$be the set of nonnegative numbers and the set of nonnegative integers, respectively. Let $1 \leqq p \leqq+\infty, s \in Z_{+}$and $\mu>-1$. In the following we assume that all functions are real-valued. For any open set $O$ in $R^{n}, L^{p}(O)$ and $H^{s}(O)$ are the usual Lebesgue and Sobolev spaces, respectively.

We denote by $L^{p}\left(0, a ; x^{\mu}\right)$ a Banach space whose elements $f(x)$ are measurable in $(0, a)$ and satisfy $x^{\mu / p} f(x) \in L^{p}(0, a)$, and the norm is defined by

$$
|f|_{L^{p}\left(0, a ; x^{\mu}\right)}=\left(\int_{0}^{a} x^{\mu}|f(x)|^{p} d x\right)^{1 / p} .
$$

In particular, $L^{2}\left(0, a ; x^{\mu}\right)$ is a Hilbert space with inner product

$$
(f, g)_{L^{2}\left(0, a ; x^{\mu}\right)}=\int_{0}^{a} x^{\mu} f(x) g(x) d x
$$


We denote by $W^{s, p}\left(0, a ; x^{\mu}\right)$ a Banach space whose elements $f(x)$ and their weighted derivatives $x^{j / p} f^{(j)}(x), j=1, \ldots, s$, belong to $L^{p}\left(0, a ; x^{\mu}\right)$, where $f^{(j)}(x)$ means the $j$-th derivative of $f(x)$. Its norm is defined by

$$
|f|_{W^{s, p}\left(0, a ; x^{\mu}\right)}=\left(\sum_{j=0}^{s} \int_{0}^{a} x^{\mu+j}\left|f^{(j)}(x)\right|^{p} d x\right)^{1 / p} .
$$

We set $H^{s}\left(0, a ; x^{\mu}\right)=W^{s, 2}\left(0, a ; x^{\mu}\right)$ that is a Hilbert space with naturally induced inner product.

Let $T>0$, and put $\Omega=(0, a) \times(0, T)$. We denote by $L^{p}\left(\Omega ; x^{\mu}\right)$ a Banach space whose elements $f(x, t)$ are measurable in $\Omega$ and satisfy $x^{\mu / p} f(x, t) \in L^{p}(\Omega)$, where the norm is defined by

$$
|f|_{L^{p}\left(\Omega ; x^{\mu}\right)}=\left(\int_{\Omega} x^{\mu}|f(x, t)|^{p} d x d t\right)^{1 / p} .
$$

We denote by $H^{s}\left(\Omega ; x^{\mu}\right)$ a Hilbert space whose elements $f(x, t)$ and their weighted derivatives $x^{j / 2} \partial_{x}^{j} \partial_{t}^{k} f(x, t), 0 \leqq j+k \leqq s$, belong to $L^{2}\left(\Omega ; x^{\mu}\right)$. Its norm is defined by

$$
|f|_{H^{s}\left(\Omega ; x^{\mu}\right)}=\left(\sum_{j+k \leqq s} \int_{\Omega} x^{\mu+j}\left(\partial_{x}^{j} \partial_{t}^{k} f(x, t)\right)^{2} d x d t\right)^{1 / 2} .
$$

$H_{0}^{1}\left(0, a ; x^{\mu}\right)$ is a subspace of $H^{1}\left(0, a ; x^{\mu}\right)$ whose elements $f(x)$ satisfy $f(a)=0$. Similarly, $H_{0}^{1}\left(\Omega ; x^{\mu}\right)$ is a subspace of $H^{1}\left(\Omega ; x^{\mu}\right)$ whose elements $f(x, t)$ satisfy $f(a, t)=0$ for a. e. $t \in I$. $K^{s}\left(0, a ; x^{\mu}\right)$ is a subspace of $H^{s}\left(0, a ; x^{\mu}\right)$ whose elements $f=f(x)$ satisfy $L_{\mu}^{j} f \in H_{0}^{1}\left(0, a ; x^{\mu}\right)$ for $j=0, \ldots,[(s-1) / 2]$. Note that

$$
\begin{gathered}
K^{0}\left(0, a ; x^{\mu}\right)=L^{2}\left(0, a ; x^{\mu}\right), \quad K^{1}\left(0, a ; x^{\mu}\right)=H_{0}^{1}\left(0, a ; x^{\mu}\right), \\
K^{2}\left(0, a ; x^{\mu}\right)=H^{2}\left(0, a ; x^{\mu}\right) \cap H_{0}^{1}\left(0, a ; x^{\mu}\right) .
\end{gathered}
$$

Let $X$ be a Banach space and $J$ an interval of $R^{1}$. We denote by $C^{j}(J ; X)$ the set of $j$-times continuously differentiable mappings of $J$ into $X$.

We are now in a position to state our main theorem. We will always assume the following condition on the initial data:

(A) $\phi$ belongs to $K^{4}\left(0, a ; x^{0}\right)$ and $\psi$ belongs to $K^{3}\left(0, a ; x^{0}\right)$.

We set $I=[0, T]$ for any fixed $0<T<+\infty$. Then our main result reads as follows.

MAIN TheOREM. Assume (A). Then IBVP (P) has a unique global solution u in $C^{2}((0, a] \times I)$. 


\section{Some properties of the operator $L_{\mu}$}

3.1. The Basic Properties of $L_{\mu}$. We recall the more general suspended string operator $L_{\mu}$ for $\mu>-1$ :

$$
L_{\mu}=-\left(\frac{x}{\mu+1} \partial_{x}^{2}+\partial_{x}\right)
$$

Needless to say, $L_{\mu}$ coincides with our differential operator $L$ for $\mu=0$. Then we have

Proposition 3.1 ([Ya1]). Let $L_{\mu}$ be as in (3.1) for $\mu>-1$. Then we have the following assertions:

(i) For $f \in K^{2}\left(0, a ; x^{\mu}\right)$ and $g \in K^{1}\left(0, a ; x^{\mu}\right)$, we have

$$
\left(L_{\mu} f, g\right)_{L^{2}\left(0, a ; x^{\mu}\right)}=\int_{0}^{a} \frac{x^{\mu+1}}{\mu+1} \partial_{x} f(x) \partial_{x} g(x) d x .
$$

(ii) $L_{\mu}$ is a positive definite and self-adjoint elliptic operator on $L^{2}\left(0, a ; x^{\mu}\right)$ with domain $D(L)=K^{2}\left(0, a ; x^{\mu}\right)$, i.e.,

$$
\left(L_{\mu} f, f\right)_{L^{2}\left(0, a ; x^{\mu}\right)} \geq 0, \quad\left(L_{\mu} f, g\right)_{L^{2}\left(0, a ; x^{\mu}\right)}=\left(f, L_{\mu} g\right)_{L^{2}\left(0, a ; x^{\mu}\right)}
$$

for $f, g \in K^{2}\left(0, a ; x^{\mu}\right)$.

(iii) For $k, \ell \in Z_{+}$, there exist constants $C_{i}, i=1,2$, depending on $k, \ell, \mu$ such that

$$
C_{1}|f|_{H^{2 k+\ell}\left(0, a ; x^{\mu}\right)} \leqq\left|L^{k} f\right|_{H^{\ell}\left(0, a ; x^{\mu}\right)} \leqq C_{2}|f|_{H^{2 k+\ell}\left(0, a ; x^{\mu}\right)}
$$

for $f \in K^{2 k+\ell}\left(0, a ; x^{\mu}\right)$.

\subsection{Imbedding theorems and Rellich type theorem}

Proposition 3.2 ([Wo-Ya]). Let $s \geqq 1$. Then we have the following assertions:

(i) $H^{s}\left(0, a ; x^{\mu}\right)$ is continuously embedded in $L^{2}\left(0, a ; x^{\mu-\delta}\right)$ for any $\delta$ with $\delta \leqq 1$ and $\delta<\mu+1$ for $\mu>-1$.

(ii) $H^{s}\left(0, a ; x^{0}\right)$ is continuously embedded in $L^{r}\left(0, a ; x^{k}\right) \cap C^{s-1}((0, a])$ for $k \in R_{+}$ and $1 \leqq r \leqq+\infty$. Moreover, we have

$$
|u|_{L^{r}\left(0, a ; x^{k}\right)} \leqq C|u|_{H^{s}\left(0, a ; x^{0}\right)}, \quad u \in H^{s}\left(0, a ; x^{0}\right),
$$

where $C$ depends on $r, a$ and is independent of $s$.

As a consequence of Proposition 3.2, we have

COROLlARY 3.3 ([Wo-Ya]). Let $s \geqq 1, k \geqq 0,1 \leqq r<+\infty$ and $\mu>-1$. Then, for $u \in H^{s}\left(\Omega ; x^{\mu}\right)$ we have

$$
|u|_{L^{r}\left(\Omega ; x^{\mu+k}\right)} \leqq C|u|_{H^{s}\left(\Omega ; x^{\mu}\right)}
$$

where $C$ depends on $r, \mu, a$ and is independent of $s, T$. 
The next lemma is the Poincaré type inequality in $H_{0}^{1}\left(0, a ; x^{\mu}\right)$.

LEMmA 3.4 ([Ya1]). Let $\mu>-1$. Then, for $u \in H_{0}^{1}\left(0, a ; x^{\mu}\right)$, we have

$$
|u|_{L^{2}\left(0, a ; x^{\mu}\right)} \leqq a\left|\partial_{x} u\right|_{L^{2}\left(0, a ; x^{\mu+1}\right)} .
$$

The next lemma is crucial in our argument.

LEMMA 3.5 ([Ya2]). Let $p \geqq 1$ and $\mu>-1$. Then, for $u \in W^{1, p}\left(0, a ; x^{\mu}\right)$ we have

$$
\begin{aligned}
& \int_{0}^{a} x^{\mu}|u(x)|^{p} d x \leqq C_{1}\left(\int_{0}^{a} x^{\mu+1}|u(x)|^{p} d x+\int_{0}^{a} x^{\mu+p}\left|u^{\prime}(x)\right|^{p} d x\right), \\
& \sup _{x \in(0, a)} x^{\mu}|u(x)|^{p} \leqq C_{2}\left(\int_{0}^{a} x^{\mu+1}|u(x)|^{p} d x+\int_{0}^{a} x^{\mu+p-1}\left|u^{\prime}(x)\right|^{p} d x\right),
\end{aligned}
$$

where the constants $C_{i}>0$ depend on $\mu, a, p$.

The following proposition is the Rellich type theorem in our weighted Sobolev space.

Proposition 3.6 ([Ya-Na-Ma]). Let $\mu>-1$. Then any bounded set of $H_{0}^{1}\left(\Omega ; x^{\mu}\right)$ is compact in $L^{2}\left(\Omega ; x^{\mu}\right)$. Moreover, any bounded set of $H_{0}^{1}\left(0, a ; x^{\mu}\right)$ is compact in $L^{2}\left(0, a ; x^{\mu}\right)$.

\section{Higher order energy estimates of $(\mathrm{P})$}

In this section we derive the higher order a priori estimates of the Galerkin approximate solutions. From now on we will always assume $\mu=0$ in our function spaces and the differential operator $L_{\mu}$ of the previous section. For simplicity, we abbreviate $L^{p}\left(0, a ; x^{0}\right), H^{s}\left(0, a ; x^{0}\right)$ and $K^{s}\left(0, a ; x^{0}\right)$ as $L^{p}, H^{s}$ and $K^{s}$, respectively, and the inner product $(\cdot, \cdot)_{L^{2}\left(0, a ; x^{0}\right)}$ and the norm $|\cdot|_{L^{2}\left(0, a ; x^{0}\right)}$ as $(\cdot, \cdot)_{L^{2}}$ and $|\cdot|_{L^{2}}$. Also we write $|\cdot|_{L^{p}\left(0, a ; x^{0}\right)}$ as $|\cdot|_{L^{p}}$. Furthermore we write

$$
\|u\|=((u, u))^{1 / 2}=\left(\int_{0}^{a} x \partial_{x} u(x)^{2} d x\right)^{1 / 2},
$$

which is the norm in $H_{0}^{1}$ and equivalent to $|\cdot|_{H^{1}}$ on account of Lemma 3.4.

Let us state key estimates by dividing into two subsections.

\subsection{Higher Order Energy Estimates of Solutions of (P)}

Proposition 4.1. Let $u=u(x, t) \in C^{\infty}\left(I ; K^{\infty}\left(0, a ; x^{0}\right)\right)$ be a solution of $(\mathrm{P})$. Then, for any $j=0,1, \ldots$, there exist constants $A_{j}$ depending on the norms $\left|\partial_{t}^{j+1} u(\cdot, 0)\right|_{L^{2}}$, $\left\|\partial_{t}^{j} u(\cdot, 0)\right\|$ and $T$, and a constant $\beta$ such that

$$
\left|\partial_{t}^{j+1} u(\cdot, t)\right|_{L^{2}}^{2}+\left\|\partial_{t}^{j} u(\cdot, t)\right\|^{2} \leqq\left(\left|\partial_{t}^{j+1} u(\cdot, 0)\right|_{L^{2}}^{2}+\left\|\partial_{t}^{j} u(\cdot, 0)\right\|^{2}+A_{j} \tau\right) e^{\beta \tau}
$$

holds on any interval $[0, \tau)$ of I. 
Proof. We use Proposition 3.1 (i) to obtain

$$
0=\left(\partial_{t}^{2} u+L u+u^{3}, \partial_{t} u\right)_{L^{2}}=\frac{1}{2} \frac{d}{d t}\left(\left|\partial_{t} u\right|_{L^{2}}^{2}+\|u\|^{2}\right)+\frac{1}{4} \frac{d}{d t}|u|_{L^{4}}^{4},
$$

which implies

$$
\begin{aligned}
\left|\partial_{t} u(\cdot, t)\right|_{L^{2}}^{2}+\|u(\cdot, t)\|^{2}+|u(\cdot, t)|_{L^{4}}^{4} & =\left|\partial_{t} u(\cdot, 0)\right|_{L^{2}}^{2}+\|u(\cdot, 0)\|^{2}+|u(\cdot, 0)|_{L^{4}}^{4} \\
& \leqq\left|\partial_{t} u(\cdot, 0)\right|_{L^{2}}^{2}+|u(\cdot, 0)|_{H^{1}}^{2}+C|u(\cdot, 0)|_{H^{1}}^{4}
\end{aligned}
$$

for all $t \in[0, \tau)$, where we used Proposition 3.2 in the last step.

Differentiating the equation in (P) $j$-times with respect to $t$, multiplying by $\partial_{t}^{j+1} u$, and integrating from 0 to $a$ with respect to $x$, we obtain

$$
\left(\partial_{t}^{j+2} u+L \partial_{t}^{j} u+\partial_{t}^{j} u^{3}, \partial_{t}^{j+1} u\right)_{L^{2}}=0,
$$

which implies

$$
\frac{d}{d t}\left(\left|\partial_{t}^{j+1} u\right|_{L^{2}}^{2}+\left\|\partial_{t}^{j} u\right\|^{2}\right)=-2\left(\partial_{t}^{j} u^{3}, \partial_{t}^{j+1} u\right)_{L^{2}} \leqq\left|\partial_{t}^{j} u^{3}\right|_{L^{2}}^{2}+\left|\partial_{t}^{j+1} u\right|_{L^{2}}^{2} .
$$

Here we can write

$$
\partial_{t}^{j} u^{3}=3 u^{2} \partial_{t}^{j} u+Q_{j}\left(u, \partial_{t} u, \ldots, \partial_{t}^{j-1} u\right),
$$

where $Q_{j}\left(X_{0}, X_{1}, \ldots, X_{j-1}\right)$ is a certain polynomial of degree 3 in variables $X_{0}, X_{1}, \ldots$, $X_{j-1}$. If we use the following inequality

$$
|f g h|_{L^{2}} \leqq C\|f\|\|g\|\|h\|, \quad f, g, h \in H_{0}^{1},
$$

then we get

$$
\begin{gathered}
\left|u^{2} \partial_{t}^{j} u\right|_{L^{2}} \leqq C\|u\|^{2}\left\|\partial_{t}^{j} u\right\| \leqq C_{0}\left\|\partial_{t}^{j} u\right\|, \\
\left|Q_{j}\left(u, \partial_{t} u, \ldots, \partial_{t}^{j-1} u\right)\right|_{L^{2}} \leqq C Q_{j}\left(\|u\|,\left\|\partial_{t} u\right\|, \ldots,\left\|\partial_{t}^{j-1} u\right\|\right),
\end{gathered}
$$

where we set

$$
C_{0}=C\left(\left|\partial_{t} u(\cdot, 0)\right|_{L^{2}}^{2}+|u(\cdot, 0)|_{H^{1}}^{2}+C|u(\cdot, 0)|_{H^{1}}^{4}\right) .
$$

The inequality (4.3) follows from Hölder's inequality, Proposition 3.2 and Lemma 3.4. Hence combining (4.2) with (4.4)-(4.5), we arrive at

$$
\frac{d}{d t}\left(\left|\partial_{t}^{j+1} u\right|^{2}+\left\|\partial_{t}^{j} u\right\|^{2}\right) \leqq\left|\partial_{t}^{j+1} u\right|_{L^{2}}^{2}+C\left\{C_{0}\left\|\partial_{t}^{j} u\right\|+Q_{j}\left(\|u\|,\left\|\partial_{t} u\right\|, \ldots,\left\|\partial_{t}^{j-1} u\right\|\right)\right\} .
$$

Since $Q_{1}=0$, it follows from (4.6) with $j=1$ that (4.1) holds for $j=1$ on account of Gronwall's lemma. Moreover, we have

$$
Q_{2}\left(\|u\|,\left\|\partial_{t} u\right\|\right) \leq C\left(\|u(0)\|,\left\|\partial_{t} u(0)\right\|\right) .
$$


Applying this bound to (4.6) and using again Gronwall's lemma, we conclude (4.1) holds for $j=2$. Thus the estimate (4.1) for $j \geqq 3$ follows from the induction argument. The proof of Proposition 4.1 is now finished.

4.2. Higher Order Estimates of Approximate Solutions. In what follows we construct the approximate solutions of (P) by using the Galerkin method (cf. [Wo-Ya]), and obtain the higher order energy estimates. For this purpose we need to impose the suitable smoothness on the initial data as in assumption (A).

Let us take the approximate solutions to $(\mathrm{P})$ of the form

$$
u_{m}(x, t)=\sum_{i=1}^{m} a_{i}^{m}(t) \phi_{i}(x), \quad m=1,2, \ldots,
$$

satisfying

$$
\left(\partial_{t}^{2} u_{m}+L u_{m}+u_{m}^{3}, \phi_{k}\right)_{L^{2}}=0, \quad k=1, \ldots, m,
$$

with the initial condition

$$
u_{m}(x, 0)=\sum_{i=1}^{m}\left(\phi, \phi_{i}\right)_{L^{2}} \phi_{i}(x), \quad \partial_{t} u_{m}(x, 0)=\sum_{i=1}^{m}\left(\psi, \phi_{i}\right)_{L^{2}} \phi_{i}(x) .
$$

Hence (4.7)-(4.8) is IVP to a system of second-order ODEs with unknown functions $a_{1}^{m}(t), \ldots, a_{m}^{m}(t)$. It follows from assumption (A) that

$$
\left|\sum_{i=1}^{m}\left(\phi, \phi_{i}\right) \phi_{i}(\cdot)-\phi(\cdot)\right|_{H^{4}} \rightarrow 0, \quad\left|\sum_{i=1}^{m}\left(\psi, \phi_{i}\right) \phi_{i}(\cdot)-\psi(\cdot)\right|_{H^{3}} \rightarrow 0 \quad \text { as } m \rightarrow+\infty .
$$

The next proposition plays a central role in our argument.

Proposition 4.2. Assume (A). Let $u_{m}$ be the solutions of (4.7)-(4.8). Then we have

$$
\left|\partial_{t}^{j+1} u_{m}(\cdot, t)\right|_{L^{2}}+\left\|\partial_{t}^{j} u_{m}(\cdot, t)\right\| \leqq C_{j+1}, \quad 0 \leqq j \leqq 3, \quad t \in I,
$$

where the constant $C_{j+1}$ depends on $T,|\phi|_{H^{j+1}}$ and $|\psi|_{H^{j}}$, but is independent of $m$.

ProOF. Differentiating equation (4.7) $j$-times with respect to $t$, multiplying by $\frac{d^{j+1}}{d t^{j+1}} a_{k}^{m}(t)$ and summing up from $k=1$ to $m$, we have

$$
\left(\partial_{t}^{j+2} u_{m}+L \partial_{t}^{j} u_{m}+\partial_{t}^{j} u_{m}^{3}, \partial_{t}^{j+1} u_{m}\right)_{L^{2}}=0 .
$$

Hence we obtain the estimate (4.1) of Proposition 4.1 for $u$ replaced by $u_{m}$, i.e.,

$$
\left|\partial_{t}^{j+1} u_{m}(\cdot, t)\right|_{L^{2}}^{2}+\left\|\partial_{t}^{j} u_{m}(\cdot, t)\right\|^{2} \leqq\left(\left|\partial_{t}^{j+1} u_{m}(\cdot, 0)\right|_{L^{2}}^{2}+\left\|\partial_{t}^{j} u_{m}(\cdot, 0)\right\|^{2}+A_{j} \tau\right) e^{\beta \tau}
$$

for $t \in[0, \tau)$. If we establish the following bounds independent of $m$,

$$
\left|\partial_{t}^{j+1} u_{m}(\cdot, 0)\right|_{L^{2}}+\left\|\partial_{t}^{j} u_{m}(\cdot, 0)\right\| \leqq C_{j+1}, \quad 0 \leqq j \leqq 3,
$$


for $m=1,2, \ldots$, then we have the required estimate (4.10). These bounds will be given in Lemmas 4.4-4.5 below.

In what follows, we pay an attention to prove (4.11). The crucial point of the treatment of our cubic nonlinear term is given by the following lemma, which states that $H^{s}$ forms an algebra for a suitable integer $s$.

LEMmA 4.3. Let $f, g, h \in K^{s}$ for some integer $1 \leqq s \leqq 4$. Then $f g h \in K^{s}$ and there exists a constant $C$ such that

$$
|f g h|_{H^{s}} \leqq C|f|_{H^{s}}|g|_{H^{s}}|h|_{H^{s}} .
$$

In particular, let $f, g \in K^{s}$ for $s=1$, 2. Then $f g \in K^{s}$ and we have

$$
|f g|_{H^{s}} \leqq C|f|_{H^{s}}|g|_{H^{s}} .
$$

Proof. Since (4.13) is an immediate consequence of (4.12), we may prove only (4.12). In the following equation

$$
|f g h|_{H^{s}}^{2}=\sum_{k=0}^{s} \int_{0}^{a} x^{k}\left|(f g h)^{(k)}\right|^{2} d x,
$$

it suffices to estimate only the term for $k=s$, since the other terms can be estimated in the similar way. We see from Leibniz rule that

$$
(f g h)^{(s)}=\sum_{j=0}^{s} \sum_{l=0}^{j} C_{j l s} f^{(j-l)} g^{(l)} h^{(s-j)} .
$$

Hence we have

$$
\begin{aligned}
\int_{0}^{a} x^{s}\left|(f g h)^{(s)}\right|^{2} d x \leqq & \sum_{j=0}^{s} \sum_{l=0}^{j} C_{j l s} \int_{0}^{a} x^{s}\left|f^{(j-l)}(x)\right|^{2}\left|g^{(l)}(x)\right|^{2}\left|h^{(s-j)}\right|^{2} d x \\
= & \int_{0}^{a}|f(x)|^{2}|g(x)|^{2} x^{s}\left|h^{(s)}(x)\right|^{2} d x \\
& +\sum_{j=1}^{s} C_{j 0 s} \int_{0}^{a} x^{j}\left|f^{(j)}(x)\right|^{2}|g(x)|^{2} x^{s-j}\left|h^{(s-j)}(x)\right|^{2} d x \\
& +\sum_{j=1}^{s} \sum_{l=1}^{j} C_{j l s} \int_{0}^{a} x^{j-l}\left|f^{(j-l)}(x)\right|^{2} \cdot x^{l}\left|g^{(l)}(x)\right| \cdot x^{s-j}\left|h^{(s-j)}(x)\right|^{2} d x .
\end{aligned}
$$

Here it follows from Lemma 3.5 that

$$
\sup _{x \in(0, a)}|f(x)|^{2} \leqq C\left(\int_{0}^{a} x|f(x)|^{2} d x+\int_{0}^{a} x\left|f^{\prime}(x)\right|^{2} d x\right) \leqq C|f|_{H^{1}}^{2},
$$


and in a similar way, we have $\sup _{x \in(0, a)}|g(x)|^{2} \leqq C|g|_{H^{1}}^{2}$. Therefore, the first term in the right-hand side of (4.14) can be estimated by

$$
\begin{aligned}
\int_{0}^{a}|f(x)|^{2}|g(x)|^{2} x^{s}\left|h^{(s)}(x)\right|^{2} d x & \leqq \sup _{x \in(0, a)}|f(x)|^{2} \sup _{x \in(0, a)}|g(x)|^{2} \int_{0}^{a} x^{s}\left|h^{(s)}(x)\right|^{2} d x \\
& \leqq C|f|_{H^{1}}^{2}|g|_{H^{1}}^{2}|h|_{H^{s}}^{2}
\end{aligned}
$$

Next we have, for $j=1, \ldots, s$,

$$
\begin{aligned}
& \sup _{x \in(0, a)} x^{s-j}\left|h^{(s-j)}(x)\right|^{2} \\
& \quad \leqq C\left(\int_{0}^{a} x^{s-j+1}\left|h^{(s-j)}(x)\right|^{2} d x+\int_{0}^{a} x^{s-j+1}\left|h^{(s-j+1)}(x)\right|^{2} d x\right) \\
& \quad \leqq C|h|_{H^{s}}^{2},
\end{aligned}
$$

and hence, the second term in the right-hand side of (4.14) can be estimated by

$$
\begin{aligned}
\sum_{j=1}^{s} & C_{j 0 s} \int_{0}^{a} x^{j}\left|f^{(j)}(x)\right|^{2}|g(x)|^{2} x^{s-j}\left|h^{(s-j)}(x)\right|^{2} d x \\
& \leqq \sum_{j=1}^{s} C_{j 0 s} \sup _{x \in(0, a)}|g(x)|^{2} \sup _{x \in(0, a)} x^{s-j}\left|h^{(s-j)}(x)\right|^{2} \int_{0}^{a} x^{j}\left|f^{(j)}(x)\right|^{2} d x \\
& \leqq C|f|_{H^{s}}^{2}|g|_{H^{1}}^{2}|h|_{H^{s}}^{2},
\end{aligned}
$$

since $\sup _{x \in(0, a)}|g(x)|^{2} \leqq C|g|_{H^{1}}^{2}$. In the similar way, since we have, for $1 \leqq l \leqq j \leqq s$,

$$
\begin{aligned}
\sup _{x \in(0, a)} x^{j-l}\left|f^{(j-l)}(x)\right|^{2} & \leqq C\left(\int_{0}^{a} x^{j-l+1}\left|f^{(j-l)}(x)\right|^{2} d x+\int_{0}^{a} x^{j-l+1}\left|f^{(j-l+1)}(x)\right|^{2} d x\right) \\
& \leqq C\left(|f|_{H^{s-1}}^{2}+|f|_{H^{s}}^{2}\right),
\end{aligned}
$$

the last term in the right-hand side of (4.14) can be estimated by

$$
\begin{aligned}
\sum_{j=1}^{s} & \sum_{l=1}^{j} C_{j l s} \int_{0}^{a} x^{j-l}\left|f^{(j-l)}(x)\right|^{2} \cdot x^{l}\left|g^{(l)}(x)\right| \cdot x^{s-j}\left|h^{(s-j)}(x)\right|^{2} d x \\
& \leqq \sum_{j=1}^{s} \sum_{l=1}^{j} C_{j l s} \sup _{x \in(0, a)} x^{j-l}\left|f^{(j-l)}(x)\right|^{2} \sup _{x \in(0, a)} x^{s-j}\left|h^{(s-j)}(x)\right|^{2} \int_{0}^{a} x^{l}\left|g^{(l)}(x)\right| d x \\
& \leqq C|f|_{H^{s}}^{2}|g|_{H^{s}}^{2}|h|_{H^{s}}^{2},
\end{aligned}
$$

where we used (4.16). Summarizing (4.14)-(4.18), we conclude the estimate (4.12). 
It can be checked that $L^{j}(f g h) \in H_{0}^{1}$ for $j=0, \ldots,[(s-1) / 2]$, provided $1 \leqq s \leqq 4$, and hence, we conclude $f g h \in K^{s}$ if $f, g, h \in K^{s}$. Finally, if $f, g \in K^{s}$ for $s=1,2$, then $f g \in K^{s}$, since $f g \in H_{0}^{1}$. The proof of Lemma 4.3 is complete.

Let us define a projector $P_{m}$ of $L^{2}\left(0, a ; x^{0}\right)$ into itself by the cut-off Bessel-Fourier series

$$
P_{m} u=\sum_{i=1}^{m} u_{i} \phi_{i}, \quad u_{i}=\left(u, \phi_{i}\right) .
$$

Note that $P_{m}$ commutes the operators $L$ and $\partial_{t}$, and satisfies $\left|P_{m} f\right|_{L^{2}} \leqq|f|_{L^{2}}$. Clearly (4.7) is equivalent to the following equation

$$
\partial_{t}^{2} u_{m}+L u_{m}+P_{m} u_{m}^{3}=0 .
$$

Differentiating (4.19) $j$-times with respect to $t$ for $0 \leqq j \leqq 2$ and putting $t=0$, we obtain

$$
\partial_{t}^{j+2} u_{m}(x, 0)=-L \partial_{t}^{j} u_{m}(x, 0)-P_{m} \partial_{t}^{j} u_{m}(x, 0)^{3} .
$$

Then putting $j=1,2$ into this equation and using (4.19) again, we have the following useful equations:

$$
\partial_{t}^{4} u_{m}(x, 0)=L^{2} u_{m}(x, 0)+L P_{m} u_{m}(x, 0)^{3}-P_{m} \partial_{t}^{2} u_{m}(x, 0)^{3} .
$$

Now we are in a position to prove (4.11) by stating the following two lemmas.

LEMma 4.4. Let $u_{m}$ be the solutions of (4.7)-(4.8). Then for $0 \leqq j \leqq 3$ we have

$$
\left|\partial_{t}^{j+1} u_{m}(\cdot, 0)\right|_{L^{2}} \leqq C_{j+1},
$$

where $C_{j+1}$ is a certain polynomial in $|\phi|_{H^{j+1}}$ and $|\psi|_{H^{j}}$, independent of $m$.

Proof. (4.22) for $j=0$ follows immediately from the energy conservation law. We divide the proof of (4.22) into three cases.

CASE: $j=1$. In this case we can use (4.19) at $t=0$, and it follows from Proposition 3.1 (iii) and (4.3) that

$$
\begin{aligned}
\left|\partial_{t}^{2} u_{m}(\cdot, 0)\right|_{L^{2}} & \leqq\left|L u_{m}(\cdot, 0)\right|_{L^{2}}+\left|u_{m}(\cdot, 0)^{3}\right|_{L^{2}} \leqq C\left(\left|u_{m}(\cdot, 0)\right|_{H^{2}}+\left\|u_{m}(\cdot, 0)\right\|^{3}\right) \\
& \leqq C\left(|\phi|_{H^{2}}+|\phi|_{H^{1}}^{3}\right)
\end{aligned}
$$

for large $m$, which proves (4.22) for $j=1$.

CASE: $j=2$. In this case, we can use (4.20), and using again Proposition 3.1 (iii) and (4.3), we have

$$
\left|\partial_{t}^{3} u_{m}(\cdot, 0)\right|_{L^{2}} \leqq\left|L \partial_{t} u_{m}(\cdot, 0)\right|_{L^{2}}+3\left|u_{m}(\cdot, 0)^{2} \partial_{t} u_{m}(\cdot, 0)\right|_{L^{2}}
$$




$$
\begin{aligned}
& \leqq C\left(\left|\partial_{t} u_{m}(\cdot, 0)\right|_{H^{2}}+\left\|u_{m}(\cdot, 0)\right\|^{2}\left\|\partial_{t} u_{m}(\cdot, 0)\right\|\right) \\
& \leqq C\left(|\psi|_{H^{2}}+|\phi|_{H^{1}}^{2}|\psi|_{H^{1}}\right)
\end{aligned}
$$

for large $m$, which proves (4.22) for $j=2$.

CASE: $j=3$. In this case, we can use (4.21), and it follows from Proposition 3.1 (iii) and (4.3) that

$$
\begin{aligned}
\left|\partial_{t}^{4} u_{m}(\cdot, 0)\right|_{L^{2}} \leqq & \left|L^{2} u_{m}(\cdot, 0)\right|_{L^{2}}+\left|L u_{m}(\cdot, 0)^{3}\right|_{L^{2}}+3\left|u_{m}(\cdot, 0)^{2} \partial_{t}^{2} u_{m}(\cdot, 0)\right|_{L^{2}} \\
& +6\left|u_{m}(\cdot, 0)\left(\partial_{t} u_{m}(\cdot, 0)\right)^{2}\right|_{L^{2}} \\
\leqq & C\left(\left|u_{m}(\cdot, 0)\right|_{H^{4}}+\left|u_{m}(\cdot, 0)^{3}\right|_{H^{2}}\right)+3\left|u_{m}(\cdot, 0)^{2} \partial_{t}^{2} u_{m}(\cdot, 0)\right|_{L^{2}} \\
& +6\left|u_{m}(\cdot, 0)\left(\partial_{t} u_{m}(\cdot, 0)\right)^{2}\right|_{L^{2}}
\end{aligned}
$$

Here we have, by Lemma 4.3,

$$
\left|u_{m}(\cdot, 0)^{3}\right|_{H^{2}} \leqq C\left|u_{m}(\cdot, 0)\right|_{H^{2}}^{3} \leqq C|\phi|_{H^{2}}^{3},
$$

and by the estimate of case $j=1$,

$$
\begin{gathered}
\left|u_{m}(\cdot, 0)^{2} \partial_{t}^{2} u_{m}(\cdot, 0)\right|_{L^{2}} \leqq\left|u_{m}(\cdot, 0)\right|_{L^{\infty}}^{2}\left|\partial_{t}^{2} u_{m}(\cdot, 0)\right|_{L^{2}} \leqq C|\phi|_{H^{1}}^{2}\left(|\phi|_{H^{2}}+|\phi|_{H^{1}}^{3}\right), \\
\left|u_{m}(\cdot, 0)\left(\partial_{t} u_{m}(\cdot, 0)\right)^{2}\right|_{L^{2}} \leqq\left|u_{m}(\cdot, 0)\right|_{L^{2}}\left|\partial_{t} u_{m}(\cdot, 0)\right|_{L^{\infty}}^{2} \leqq C|\phi|_{L^{2}}|\psi|_{H^{1}}^{2} .
\end{gathered}
$$

Combining the estimates obtained now, we arrive at the estimate (4.22) for $j=3$. The proof of Lemma 4.4 is complete.

LEMmA 4.5. Let $u_{m}$ be the solutions of (4.7)-(4.8). Then for $0 \leqq j \leqq 3$ we have

$$
\left\|\partial_{t}^{j} u_{m}(\cdot, 0)\right\| \leqq C_{j+1},
$$

where each $C_{j+1}$ is a certain polynomial of $|\phi|_{H^{j+1}}$ and $|\psi|_{H^{j}}$, but is independent of $m$.

ProOf. We note that there exists a constant $C>1$ such that

$$
C^{-1}\|u\| \leqq\left|L^{1 / 2} u\right|_{L^{2}} \leqq C\|u\|
$$

holds for any $u \in K^{1}$, which can be easily proved by using the spectral representation of $L$ (see Appendix).

Since (4.23) for $j=0,1$ is trivial, it suffices to prove the cases $j=2$, 3. In what follows we will use Lemma 4.3 without any comment.

THE CASE: $j=2$. Using (4.19) and (4.24), we have

$$
\begin{aligned}
\left\|\partial_{t}^{2} u_{m}(\cdot, 0)\right\| & \leqq C\left(\left|L^{3 / 2} u_{m}(\cdot, 0)\right|_{L^{2}}+\left|L^{1 / 2} u_{m}(\cdot, 0)^{3}\right|_{L^{2}}\right) \\
& \leqq C\left(\left|u_{m}(\cdot, 0)\right|_{H^{3}}+\left|u_{m}(\cdot, 0)\right|_{H^{1}}^{3}\right) \\
& \leqq C\left(|\phi|_{H^{3}}+|\phi|_{H^{1}}^{3}\right)
\end{aligned}
$$

which proves (4.23) for $j=2$. 
THE CASE: $j=3$. Using (4.20) and (4.24), we have

$$
\begin{aligned}
\left\|\partial_{t}^{3} u_{m}(\cdot, 0)\right\| & \leqq C\left(\left|L^{3 / 2} \partial_{t} u_{m}(\cdot, 0)\right|_{L^{2}}+\left|L^{1 / 2} \partial_{t} u_{m}(\cdot, 0)^{3}\right|_{L^{2}}\right) \\
& \leqq C\left(\left|\partial_{t} u_{m}(\cdot, 0)\right|_{H^{3}}+\left|\partial_{t} u_{m}(\cdot, 0)^{3}\right|_{H^{1}}\right) \\
& \leqq C\left(\left|\partial_{t} u_{m}(\cdot, 0)\right|_{H^{3}}+\left|u_{m}(\cdot, 0)^{2} \partial_{t} u_{m}(\cdot, 0)\right|_{H^{1}}\right) \\
& \leqq C\left(\left|\partial_{t} u_{m}(\cdot, 0)\right|_{H^{3}}+\left|u_{m}(\cdot, 0)\right|_{H^{1}}^{2}\left|\partial_{t} u_{m}(\cdot, 0)\right|_{H^{1}}\right) \\
& \leqq C\left(|\psi|_{H^{3}}+|\phi|_{H^{1}}^{2}|\psi|_{H^{1}}\right),
\end{aligned}
$$

which proves (4.23) for $j=3$. The proof of Lemma 4.5 is now finished.

\section{Proof of Main Theorem}

Recalling the abbreviation $H^{s}=H^{s}\left(0, a ; x^{0}\right)$ and $W^{s, p}=W^{s, p}\left(0, a ; x^{0}\right)$, we shall prove here the following.

THEOREM 5.1. Suppose assumption (A). Then (P) has a unique solution u satisfying

$$
u \in C^{3}\left(I ; L^{2}\right) \bigcap_{j=0}^{2} C^{j}\left(I ; H^{3-j} \cap H_{0}^{1}\right) .
$$

The following remark is important.

REMARK 5.2. In addition to the result of Theorem 5.1, it should be mentioned that our problem (P) is $H^{4}$ well-posed. More precisely, the solution $u$ from Theorem 5.1 also satisfies

$$
u \in W^{4, \infty}\left(I ; L^{2}\right) \bigcap_{j=0}^{3} W^{j, \infty}\left(I ; H^{4-j} \cap H_{0}^{1}\right) .
$$

The proof of Theorem 5.1 will be done together with the proof of (5.1).

\subsection{Compactness of Approximate Solutions}

LEMMA 5.2. Let $u_{m}$ be the approximate solutions of (4.7)-(4.8). Then there exists a limiting function $u$ of $\left\{u_{m}\right\}$ along a subsequence such that, for $0 \leqq j \leqq 3$,

$$
\begin{aligned}
& \partial_{t}^{j} u \in H_{0}^{1}\left(\Omega ; x^{0}\right) \cap L^{\infty}\left(I ; H_{0}^{1}\left(0, a ; x^{0}\right)\right), \quad \partial_{t}^{4} u \in L^{2}\left(\Omega ; x^{0}\right) \cap L^{\infty}\left(I ; L^{2}\left(0, a ; x^{0}\right)\right), \\
& \partial_{t}^{j} u_{m} \rightarrow \partial_{t}^{j} u \quad \text { weakly in } H_{0}^{1}\left(\Omega ; x^{0}\right), \\
& \partial_{t}^{j} u_{m} \rightarrow \partial_{t}^{j} u \quad \text { weakly* in } L^{\infty}\left(I ; H_{0}^{1}\left(0, a ; x^{0}\right)\right), \\
& \partial_{t}^{j} u_{m} \rightarrow \partial_{t}^{j} u \quad \text { strongly in } L^{2}\left(\Omega ; x^{0}\right), \\
& \partial_{t}^{j} u_{m} \rightarrow \partial_{t}^{j} u \quad \text { strongly in } L^{\infty}\left(I ; L^{2}\left(0, a ; x^{0}\right)\right),
\end{aligned}
$$




$$
\begin{aligned}
& \partial_{t}^{j} u_{m}(x, t) \rightarrow \partial_{t}^{j} u(x, t) \quad \text { a.e. in } \Omega, \\
& \partial_{t}^{4} u_{m} \rightarrow \partial_{t}^{4} u \quad \text { weakly in } L^{2}\left(\Omega ; x^{0}\right), \\
& \partial_{t}^{4} u_{m} \rightarrow \partial_{t}^{4} u \quad \text { weakly } \text { in } L^{\infty}\left(I ; L^{2}\left(0, a ; x^{0}\right)\right),
\end{aligned}
$$

where each derivative $\partial_{t}^{j} u$ is taken in the distributional sense.

Proof. Since $\left\{\partial_{t}^{j} u_{m}\right\}(j=0, \ldots, 3)$ and $\left\{\partial_{t}^{4} u_{m}\right\}$ are uniformly bounded in $H_{0}^{1}\left(\Omega ; x^{0}\right)$ and $L^{2}\left(\Omega ; x^{0}\right)$, respectively, on account of Proposition 4.2, it follows from Proposition 3.6 that there exists a limiting function $w_{j}$ of $\left\{\partial_{t}^{j} u_{m}\right\}$ along a subsequence such that, for $0 \leqq j \leqq$ 3 ,

$$
\begin{aligned}
& \partial_{t}^{j} u_{m} \rightarrow w_{j} \quad \text { weakly in } H_{0}^{1}\left(\Omega ; x^{0}\right), \\
& \partial_{t}^{j} u_{m} \rightarrow w_{j} \quad \text { weakly* in } L^{\infty}\left(I ; H_{0}^{1}\left(0, a ; x^{0}\right)\right), \\
& \partial_{t}^{j} u_{m} \rightarrow w_{j} \quad \text { strongly in } L^{2}\left(\Omega ; x^{0}\right), \\
& \partial_{t}^{j} u_{m} \rightarrow w_{j} \quad \text { strongly in } L^{\infty}\left(I ; L^{2}\left(0, a ; x^{0}\right)\right), \\
& \partial_{t}^{4} u_{m} \rightarrow w_{4} \quad \text { weakly in } L^{2}\left(\Omega ; x^{0}\right), \\
& \partial_{t}^{4} u_{m} \rightarrow w_{4} \quad \text { weakly* in } L^{\infty}\left(I ; L^{2}\left(0, a ; x^{0}\right)\right),
\end{aligned}
$$

and hence, each $w_{j}(j=0, \ldots, 3)$ belongs to $L^{\infty}\left(I ; H_{0}^{1}\left(0, a ; x^{0}\right)\right)$ and further, $w_{4}$ belongs to $L^{\infty}\left(I ; L^{2}\left(0, a ; x^{0}\right)\right)$. Put $u=w_{0}$. Then it can be readily checked that $\partial_{t}^{j} u=w_{j}$ for $j=0, \ldots, 4$ by the standard distributional argument. Finally, as is well-known, since the convergent sequence in $L^{\infty}\left(I ; L^{2}\left(0, a ; x^{0}\right)\right)$ has a convergent subsequence a.e. in $\Omega$, it follows that each $\partial_{t}^{j} u_{m}(x, t)$ converges to $\partial_{t}^{j} u(x, t)$ a.e. in $\Omega$. Thus Lemma 5.2 is proved.

The following lemma gives the uniform convergence of $\left\{\partial_{t}^{j} u_{m}(\cdot, t)\right\}$ with respect to $t$.

LEMMA 5.3. Let $u_{m}$ be as in Lemma 5.2. Then the limiting function $\partial_{t}^{j} u$ of Lemma 5.2 belongs to $C\left(I ; H_{0}^{1}\left(0, a ; x^{0}\right)\right)$ for $j=0,1,2$ and $\partial_{t}^{3} u$ belongs to $C\left(I ; L^{2}\left(0, a ; x^{0}\right)\right)$, and there exists a subsequence, denoted by the same, of $\left\{u_{m}\right\}$ such that

$$
\sup _{t \in I}\left|\partial_{t}^{j} u_{m}(\cdot, t)-\partial_{t}^{j} u(\cdot, t)\right|_{L^{2}} \rightarrow 0 \quad \text { for } 0 \leqq j \leqq 3 \text { as } m \rightarrow+\infty .
$$

Proof. Since the sequence $\left\{\partial_{t}^{j} u_{m}(\cdot, t)\right\}$ is uniformly bounded on $I$ in $H_{0}^{1}$ for $j=$ $0,1,2$, or even $j=3$ by Proposition 4.2, we have, for $j=0,1,2$.

$$
\left|\left(\left(\partial_{t}^{j} u_{m}\left(\cdot, t_{1}\right)-\partial_{t}^{j} u_{m}\left(\cdot, t_{2}\right), \rho\right)\right)\right| \leqq\left|\int_{t_{1}}^{t_{2}}\right|\left(\left(\partial_{\tau}^{j+1} u_{m}(\cdot, \tau), \rho\right)\right)|d \tau| \leqq C_{j+2}\left|t_{1}-t_{2}\right|\|\rho\|
$$


for all $\rho \in H_{0}^{1}$ and all $t_{1}, t_{2} \in I$. Letting $m \rightarrow+\infty$ in (5.3), we have

$$
\left|\left(\left(\partial_{t}^{j} u\left(\cdot, t_{1}\right)-\partial_{t}^{j} u\left(\cdot, t_{2}\right), \rho\right)\right)\right| \leqq C_{j+2}\left|t_{1}-t_{2}\right|\|\rho\|
$$

for all $\rho \in H_{0}^{1}$ and all $t_{1}, t_{2} \in I$, which is ensured by Lemma 5.2. Thus we get, by the Riesz theorem,

$$
\left\|\partial_{t}^{j} u\left(\cdot, t_{1}\right)-\partial_{t}^{j} u\left(\cdot, t_{2}\right)\right\| \leqq C_{j+2}\left|t_{1}-t_{2}\right|,
$$

which proves $\partial_{t}^{j} u \in C\left(I ; H_{0}^{1}\right)$ for $j=0,1,2$.

Next, the sequence $\left\{\partial_{t}^{j} u_{m}(\cdot, t)\right\}$ is uniformly bounded on $I$ in $L^{2}\left(0, a ; x^{0}\right)$ for $j=$ $0,1,2,3$, or even $j=4$. Furthermore, it is also equi-continuous on $I$ in $L^{2}\left(0, a ; x^{0}\right)$ for $j=0,1,2,3$. In fact, we have, for $j=0,1,2,3$,

$$
\left|\partial_{t}^{j} u_{m}\left(\cdot, t_{1}\right)-\partial_{t}^{j} u_{m}\left(\cdot, t_{2}\right)\right|_{L^{2}} \leqq\left.\left|\int_{t_{2}}^{t_{1}}\right| \partial_{\tau}^{j+1} u_{m}(\cdot, \tau)\right|_{L^{2}} d \tau\left|\leqq C_{j+1}\right| t_{1}-t_{2} \mid
$$

for all $t_{1}, t_{2} \in I$. Therefore, using the Ascoli-Arzelà theorem, we can choose a subsequence, denoted by the same, of $\left\{\partial_{t}^{j} u_{m}(\cdot, t)\right\}$ such that it converges uniformly to $\partial_{t}^{j} u \in$ $C\left(I ; L^{2}\left(0, a ; x^{0}\right)\right)$ on $I$ strongly in $L^{2}\left(0, a ; x^{0}\right)$. This proves (5.2). The proof of Lemma 5.3 is complete.

We need the (uniform) convergence of $\partial_{t}^{j} u_{m}$ in $L^{2}\left(0, a ; x^{0}\right)$ up to $j=2$.

LEMMA 5.4. Let $u_{m}$ and $u$ be as in Lemma 5.3. Then we have $\partial_{t}^{j} u^{3} \in$ $C\left(I ; H_{0}^{1}\left(0, a ; x^{0}\right)\right)$ for $0 \leqq j \leqq 2$ and

$$
\sup _{t \in I}\left|\partial_{t}^{j} u_{m}^{3}(\cdot, t)-\partial_{t}^{j} u^{3}(\cdot, t)\right|_{L^{2}} \rightarrow 0 \quad \text { for } 0 \leqq j \leqq 2 \quad \text { as } m \rightarrow+\infty .
$$

PROOF. Noting

$$
\partial_{t} u^{3}=3 u^{2} \partial_{t} u, \quad \partial_{t}^{2} u^{3}=6 u\left(\partial_{t} u\right)^{2}+3 u^{2} \partial_{t}^{2} u,
$$

we can apply Lemma 4.3 to each term in the right-hand sides, since $\partial_{t}^{j} u(j=0,1,2)$ belongs to $C\left(I ; H_{0}^{1}\right)$ by Lemma 5.3. Then we get $\partial_{t}^{j} u^{3} \in C\left(I ; H_{0}^{1}\right)$ for $j=0,1,2$.

We note that there exists a constant $C_{j+1}$ depending on the norms $|\phi|_{H^{j+1}}$ and $|\psi|_{H^{j}}$ such that

$$
\sup _{\substack{(x, t) \in \Omega \\ m=1,2, \ldots}}\left|\partial_{t}^{j} u_{m}(x, t)\right| \leqq C_{j+1}, \quad j=0,1,2,
$$

$$
\sup _{t \in I}\left\|\partial_{t}^{j} u(\cdot, t)\right\| \leqq C_{j+1}, \quad j=0,1,2 .
$$


The estimate (5.5) follows from Proposition 3.2 (ii), Lemma 3.4 and Proposition 4.2 as follows:

$$
\sup _{x \in(0, a]}\left|\partial_{t}^{j} u_{m}(x, t)\right| \leqq C\left\|\partial_{t}^{j} u_{m}(\cdot, t)\right\| \leqq C_{j+1},
$$

and further, the estimates (5.6) follows from

$$
\left\|\partial_{t}^{j} u(\cdot, t)\right\| \leqq \liminf _{m \rightarrow+\infty}\left\|\partial_{t}^{j} u_{m}(\cdot, t)\right\| \leqq C_{j+1} .
$$

We prove (5.4) for each $j$ by using Proposition 4.2, Lemmas 4.3 and 5.3, and the estimates (5.5)-(5.6) without any comment.

CASE: $j=0$. It follows that

$$
\begin{aligned}
\left|u_{m}(\cdot, t)^{3}-u(\cdot, t)^{3}\right|_{L^{2}} & \leqq\left|u_{m}(\cdot, t)-u(\cdot, t)\right|_{L^{2}}\left(\left\|u_{m}(\cdot, t)\right\|^{2}+\left\|u_{m}(\cdot, t)\right\|\|u(\cdot, t)\|+\|u(\cdot, t)\|^{2}\right) \\
& \leqq C\left|u_{m}(\cdot, t)-u(\cdot, t)\right|_{L^{2}} \rightarrow 0, \\
& \text { uniformly in } t \in I, \quad m \rightarrow+\infty,
\end{aligned}
$$

which proves (5.4) for $j=0$.

CASE: $j=1$. It follows that

$$
\begin{aligned}
\left|\partial_{t} u_{m}(\cdot, t)^{3}-\partial_{t} u(\cdot, t)^{3}\right|_{L^{2}} \\
\quad \leqq C\left(\mid\left(u_{m}(\cdot, t)^{2}-\left.\left(u(\cdot, t)^{2}\right) \partial_{t} u_{m}(\cdot, t)\right|_{L^{2}}+\left|u(\cdot, t)^{2}\left(\partial_{t} u_{m}(\cdot, t)-\partial_{t} u(\cdot, t)\right)\right|_{L^{2}}\right)\right. \\
\quad \leqq C\left(\left|u_{m}(\cdot, t)-u(\cdot, t)\right|_{L^{2}}+\left|\partial_{t} u_{m}(\cdot, t)-\partial_{t} u(\cdot, t)\right|_{L^{2}}\right) \rightarrow 0,
\end{aligned}
$$

uniformly in $t \in I$ as $m \rightarrow+\infty$, which proves (5.4) for $j=1$.

CASE: $\quad j=2$. In this case, noting

$$
\begin{aligned}
\partial_{t}^{2} u_{m}^{3}-\partial_{t}^{2} u^{3}= & 6\left(u_{m}-u\right)\left(\partial_{t} u_{m}\right)^{2}+6 u\left\{\left(\partial_{t} u_{m}\right)^{2}-\left(\partial_{t} u\right)^{2}\right\} \\
& +3 u_{m}^{2}\left(\partial_{t}^{2} u_{m}-\partial_{t}^{2} u\right)+3\left(\partial_{t}^{2} u_{m}^{2}-\partial_{t}^{2} u^{2}\right),
\end{aligned}
$$

we have

$$
\begin{aligned}
\left|\partial_{t}^{2} u_{m}(\cdot, t)^{3}-\partial_{t}^{2} u(\cdot, t)^{3}\right|_{L^{2}} & \\
\leqq & C\left(\left|u_{m}(\cdot, t)-u(\cdot, t)\right|_{L^{2}}+\left|\partial_{t} u_{m}(\cdot, t)-\partial_{t} u(\cdot, t)\right|_{L^{2}}\right. \\
& \left.+\left|\partial_{t}^{2} u_{m}(\cdot, t)-\partial_{t}^{2} u(\cdot, t)\right|_{L^{2}}\right) \rightarrow 0,
\end{aligned}
$$

uniformly in $t \in I$ as $m \rightarrow+\infty$, which proves (5.4) for $j=2$. The proof of Lemma 5.4 is complete.

5.2. Proof of Theorem 5.1 and Remark 5.2. We differentiate (4.7) $j$-times with respect to $t \in I$ for $0 \leqq j \leqq 2$, apply Proposition 3.1 (i) and let $m \rightarrow+\infty$. Then using Lemmas 5.2-5.4, we obtain

$$
\left(\partial_{t}^{j+2} u(\cdot, t), \phi_{k}\right)_{L^{2}}+\left(\left(\partial_{t}^{j} u(\cdot, t), \phi_{k}\right)\right)+\left(\partial_{t}^{j} u(\cdot, t)^{3}, \phi_{k}\right)_{L^{2}}=0, \quad k=1,2, \ldots
$$


Since the linear hull generated by $\left\{\phi_{k}\right\}$ is dense in $H_{0}^{1}\left(0, a ; x^{0}\right)$, we obtain

$$
\left(\partial_{t}^{j+2} u(\cdot, t), v\right)_{L^{2}}+\left(\left(\partial_{t}^{j} u(\cdot, t), v\right)\right)+\left(\partial_{t}^{j} u(\cdot, t)^{3}, v\right)_{L^{2}}=0
$$

for all $v \in H_{0}^{1}\left(0, a ; x^{0}\right)$. This means that $\partial_{t}^{j} u$ is a weak solution of (P).

To gain the regularity in space we need the following elliptic regularity theorem of Nirenberg type.

Proposition 5.5. Let $s \in Z_{+}$and $\mu>-1$. Assume that

either $g \in H^{s}\left(0, a ; x^{\mu}\right) \cap H_{0}^{1}\left(0, a ; x^{\mu}\right)$ for $s \in N$, or $g \in L^{2}\left(0, a ; x^{\mu}\right)$ for $s=0$.

Then the elliptic BVP

$$
L_{\mu} w=g, \quad w(a)=0
$$

has a unique solution $w$ in $H^{s+2}\left(0, a ; x^{\mu}\right) \cap H_{0}^{1}\left(0, a ; x^{\mu}\right)$ satisfying

$$
|w|_{H^{s+2}\left(0, a ; x^{\mu}\right)} \leqq C|g|_{H^{s}\left(0, a ; x^{\mu}\right)},
$$

where $C$ is a constant depending on $\mu, s$.

The proof of Proposition 5.5 is postponed and will be given in Appendix.

We have already known from Lemma 5.2 that $u \in W^{4, \infty}\left(I ; L^{2}\right) \cap W^{3, \infty}\left(I ; H_{0}^{1}\right) . u \in$ $C^{3}\left(I ; L^{2}\right)$ follows from Lemma 5.3. Hence it is sufficient to prove $\partial_{t}^{j} u \in C\left(I ; H^{3-j} \cap H_{0}^{1}\right) \cap$ $L^{\infty}\left(I ; H^{4-j} \cap H_{0}^{1}\right)$ for $0 \leqq j \leqq 2$.

At first we prove $u \in C\left(I ; H^{3} \cap H_{0}^{1}\right)$. From (5.7) with $j=0$, it follows that $w \equiv u(\cdot, t)$ is a weak solution of elliptic BVP

$$
L w=g_{1}=-\left(\partial_{t}^{2} u(\cdot, t)+u(\cdot, t)^{3}\right), \quad w(a)=0 .
$$

Applying Lemmas 5.3-5.4 to $g_{1}$ in (5.9), we have $g_{1} \in C\left(I ; H_{0}^{1}\right)$. Hence we conclude from Proposition 5.5 with $s=0$ that $u(\cdot, t) \in H^{3} \cap H_{0}^{1}$ and

$$
\left|u\left(\cdot, t_{1}\right)-u\left(\cdot, t_{2}\right)\right|_{H^{3}} \leqq C\left\|g_{1}\left(\cdot, t_{1}\right)-g_{1}\left(\cdot, t_{2}\right)\right\| \quad \text { for all } t_{1}, t_{2} \in I .
$$

This proves $u \in C\left(I ; H^{3} \cap H_{0}^{1}\right)$.

Next we prove $u \in C^{1}\left(I ; H^{2} \cap H_{0}^{1}\right) \cap W^{1, \infty}\left(I ; H^{3} \cap H_{0}^{1}\right)$. It follows from (5.7) with $j=1$ that $w \equiv \partial_{t} u(\cdot, t)$ is a weak solution of elliptic BVP

$$
L w=g_{3}=-\left(\partial_{t}^{3} u(\cdot, t)+\partial_{t} u(\cdot, t)^{3}\right), \quad w(a)=0 .
$$

Since $\partial_{t}^{3} u \in C\left(I ; L^{2}\right) \cap L^{\infty}\left(I ; H_{0}^{1}\right)$ by Lemmas 5.2-5.3, and since $\partial_{t} u^{3} \in C\left(I ; L^{2}\right) \cap$ $L^{\infty}\left(I ; H_{0}^{1}\right)$ by Lemmas 4.3, 5.2 and 5.4 , we have $g_{3} \in C\left(I ; L^{2}\right) \cap L^{\infty}\left(I ; H_{0}^{1}\right)$. Thus it follows from Proposition 5.5 with $s=1$ that $\partial_{t} u \in H^{3} \cap H_{0}^{1}$ and

$$
\left|\partial_{t} u\left(\cdot, t_{1}\right)-\partial_{t} u\left(\cdot, t_{2}\right)\right|_{H^{2}} \leqq C_{2}\left|g_{3}\left(\cdot, t_{1}\right)-g_{3}\left(\cdot, t_{2}\right)\right|_{L^{2}} \quad \text { for all } t_{1}, t_{2} \in I,
$$


which proves $\partial_{t} u \in C\left(I ; H^{2} \cap H_{0}^{1}\right) \cap L^{\infty}\left(I ; H^{3} \cap H_{0}^{1}\right)$, hence $u \in C^{1}\left(I ; H^{2} \cap H_{0}^{1}\right) \cap$ $W^{1, \infty}\left(I ; H^{3} \cap H_{0}^{1}\right)$.

To see $u \in C^{2}\left(I ; H_{0}^{1}\right)$, we prepare, using the equation of (P),

$$
\begin{aligned}
\left\|\partial_{t}^{2} u\left(\cdot, t_{1}\right)-\partial_{t}^{2} u\left(\cdot, t_{2}\right)\right\| & \leqq\left\|L u\left(\cdot, t_{1}\right)-L u\left(\cdot, t_{2}\right)\right\|+\left\|u\left(\cdot, t_{1}\right)^{3}-u\left(\cdot, t_{2}\right)^{3}\right\| \\
& \leqq C\left(\left|u\left(\cdot, t_{1}\right)-u\left(\cdot, t_{2}\right)\right|_{H^{2}}+\left\|u\left(\cdot, t_{1}\right)-u\left(\cdot, t_{2}\right)\right\|\right)
\end{aligned}
$$

for all $t_{1}, t_{2} \in I$. Then it follows from the continuity of $u$ on $I$ in $H^{2}$ that $u$ belongs to $C^{2}\left(I ; H_{0}^{1}\right)$.

Now we prove $u \in W^{2, \infty}\left(I ; H^{2} \cap H_{0}^{1}\right)$. It follows from (5.7) with $j=2$ that $w \equiv$ $\partial_{t}^{2} u(\cdot, t)$ is a weak solution of elliptic BVP

$$
L w=g_{2}=-\left(\partial_{t}^{4} u(\cdot, t)+\partial_{t}^{2} u(\cdot, t)^{3}\right), \quad w(a)=0 .
$$

Since $g_{2} \in L^{\infty}\left(I ; L^{2}\right)$ by Lemmas 5.2 and 5.4, we conclude from Proposition 5.5 with $s=0$ that $\partial_{t}^{2} u(\cdot, t) \in L^{\infty}\left(I ; H^{2} \cap H_{0}^{1}\right)$, hence, $u \in W^{2, \infty}\left(I ; H^{2} \cap H_{0}^{1}\right)$.

It remains to prove $u \in L^{\infty}\left(I ; H^{4} \cap H_{0}^{1}\right)$. Let us go back to (5.9). Since $\partial_{t}^{2} u \in$ $L^{\infty}\left(I ; H^{2} \cap H_{0}^{1}\right)$ by the above argument, it follows from Lemma 4.3 that $u^{3} \in L^{\infty}\left(I ; H^{2} \cap\right.$ $\left.H_{0}^{1}\right)$, and hence, $g_{1} \in L^{\infty}\left(I ; H^{2} \cap H_{0}^{1}\right)$. Then applying again Proposition 5.5 with $s=2$ to (5.9), we have $u \in L^{\infty}\left(I ; H^{4} \cap H_{0}^{1}\right)$.

Summarizing the above argument, we conclude that the weak solution $u$ of $(\mathrm{P})$ is, in fact, the strong solution and satisfies

$$
u \in C^{3}\left(I ; L^{2}\right) \bigcap_{j=0}^{2} C^{j}\left(I ; H^{3-j} \cap H_{0}^{1}\right), \quad u \in W^{4, \infty}\left(I ; L^{2}\right) \bigcap_{j=0}^{3} W^{j, \infty}\left(I ; H^{4-j} \cap H_{0}^{1}\right) .
$$

The proof of uniqueness is standard. For the completeness we give it. Let $u, v$ be two solutions of $(\mathrm{P})$ with the same initial-boundary condition. Put $U=u-v$. Then $U$ satisfies the following problem:

$$
\partial_{t}^{2} U+L U+u^{3}-v^{3}=0, \quad U(x, 0)=\partial_{t} U(x, 0)=0, \quad U(a, t)=0 .
$$

Multiplying (5.10) by $\partial_{t} U$ and integrating, we get

$$
\frac{1}{2} \frac{d}{d t}\left(|U(t)|_{L^{2}}^{2}+\|U(t)\|^{2}\right)=-\left(u(t)^{3}-v(t)^{3}, \partial_{t} U(t)\right)_{L^{2}} .
$$

Since

$$
\begin{aligned}
\left(u(t)^{3}-v(t)^{3}, \partial_{t} U(t)\right)_{L^{2}} & \leqq \int_{0}^{a}\left|u(t)^{3}-v(t)^{3} \| \partial_{t} U(t)\right| d x \\
& \leqq C\left(|u(t)|_{L^{\infty}}^{2}+|v(t)|_{L^{\infty}}^{2}\right) \int_{0}^{a}\left|U(t) \| \partial_{t} U(t)\right| d x \\
& \leqq C\|U(t)\|\left|\partial_{t} U(t)\right|_{L^{2}},
\end{aligned}
$$


we have

$$
\frac{1}{2} \frac{d}{d t}\left(|U(t)|_{L^{2}}^{2}+\|U(t)\|^{2}\right) \leqq C\|U(t)\|\left|\partial_{t} U(t)\right|_{L^{2}} .
$$

By Gronwall's lemma, we conclude that $|U(t)|_{L^{2}}^{2}+\|U(t)\|^{2}=0$ for all $t \geq 0$, i.e., $u(t)=$ $v(t)$. Thus Theorem 5.1 and Remark 5.2 are proved.

Proof of MAIN Theorem COMPLETED. For the proof of our main theorem it suffices to prove the following.

LEMMA 5.6. $X_{3} \equiv C^{3}\left(I ; L^{2}\right) \bigcap_{j=1}^{3} C^{3-j}\left(I ; H^{j} \cap H_{0}^{1}\right)$ is embedded in $C^{2}((0, a] \times I)$.

Proof. Let $u \in X_{3}$, i.e., $u \in C^{j}\left(I ; H^{3-j} \cap H_{0}^{1}\right)$ for $j=1,2,3$, and $u \in C^{3}\left(I ; L^{2}\right)$. Then we prove that each $\partial_{x}^{\alpha} \partial_{t}^{j} u(x, t), 0 \leqq \alpha+j \leqq 2$, coincides with the derivatives in the classical sense.

Let $\delta>0$ be any fixed constant. Then $H^{s}\left(\delta, a ; x^{0}\right)$, which is defined by the same way as $H^{s}$, is isomorphic to the usual Sobolev space $H^{s}(\delta, a)$. By the Sobolev imbedding theorems, fixing $t \in I$, we can claim that $\partial_{x}^{\alpha} \partial_{t}^{j} u(x, t)$ is continuous on the interval $[\delta, a]$ of $x$ for $0 \leqq j \leqq k, 0 \leqq \alpha \leqq 2-k$. Hence $\partial_{x}^{\alpha} \partial_{t}^{j} u(x, t)$ is also continuous on $(0, a]$ for any fixed $t \in I$ on account of the arbitrariness of $\delta$. Furthermore, by the usual Sobolev inequality we have

$$
\left|\partial_{x}^{\alpha} \partial_{t}^{j} u(x, t)\right| \leqq C_{\delta}\left|\partial_{t}^{j} u(\cdot, t)\right|_{H^{3-k}\left(\delta, a ; x^{0}\right)} \leqq C_{\delta}\left|\partial_{t}^{j} u(\cdot, t)\right|_{H^{3-k}},
$$

for all $(x, t) \in[\delta, a] \times I$ and $0 \leqq j \leqq k, 0 \leqq \alpha \leqq 2-k$, where $C_{\delta}>0$ depends on $\delta$. Moreover $\partial_{x}^{\alpha} \partial_{t}^{j} u(x, t)$ is continuous in $x \in[\delta, a]$ for any fixed $t \in I$. Using (5.11), we have

$$
\begin{aligned}
& \left|\frac{1}{h}\left(\partial_{x}^{\alpha} \partial_{t}^{j-1} u(x, t+h)-\partial_{x}^{\alpha} \partial_{t}^{j-1} u(x, t)\right)-\partial_{x}^{\alpha} \partial_{t}^{j} u(x, t)\right| \\
& \quad \leq C_{\delta}\left|\frac{1}{h}\left(\partial_{t}^{j-1} u(\cdot, t+h)-\partial_{t}^{j-1} u(\cdot, t)\right)-\partial_{t}^{j} u(\cdot, t)\right|_{H^{3-k}} \rightarrow 0 \quad(h \rightarrow 0) .
\end{aligned}
$$

Therefore $\partial_{x}^{\alpha} \partial_{t}^{j} u(x, t)$ is a classical derivative with respect to $(x, t) \in[\delta, a] \times I$ for $0 \leqq$ $j \leqq k, 0 \leqq \alpha \leqq 2-k$. Since $\delta$ is arbitrary, $\partial_{x}^{\alpha} \partial_{t}^{j} u(x, t)$ is a classical derivative in $(x, t) \in$ $(0, a] \times I$.

Finally we prove that each $\partial_{x}^{\alpha} \partial_{t}^{j} u(x, t)$ is continuous on $(0, a] \times I$. Let $\left(x_{0}, t_{0}\right) \in(0, a] \times$ $I$ be arbitrarily fixed. Take $\delta \in\left(0, x_{0}\right)$. Notice that, for $(x, t) \in[\delta, a] \times I$,

$$
\begin{aligned}
& \left|\partial_{x}^{\alpha} \partial_{t}^{j} u(x, t)-\partial_{x}^{\alpha} \partial_{t}^{j} u\left(x_{0}, t_{0}\right)\right| \\
& \quad \leqq\left|\partial_{x}^{\alpha} \partial_{t}^{j} u(x, t)-\partial_{x}^{\alpha} \partial_{t}^{j} u\left(x, t_{0}\right)\right|+\left|\partial_{x}^{\alpha} \partial_{t}^{j} u\left(x, t_{0}\right)-\partial_{x}^{\alpha} \partial_{t}^{j} u\left(x_{0}, t_{0}\right)\right| .
\end{aligned}
$$

Then the first term of the right-hand side tends to 0 as $t \rightarrow t_{0}$, since $\partial_{x}^{\alpha} \partial_{t}^{j} u(x, t)$ is continuous on $I$ for any fixed $x \in(0, a]$ by (5.11). Since we have already known that $\partial_{x}^{\alpha} \partial_{t}^{j} u(x, t)$ is 
continuous on $(0, a]$ for any fixed $t \in I$, the second term of the right-hand side tends to 0 as $x \rightarrow x_{0}$. Thus $\partial_{x}^{\alpha} \partial_{t}^{j} u(x, t)$ is continuous at $\left(x_{0}, t_{0}\right)$. In conclusion, $\partial_{x}^{\alpha} \partial_{t}^{j} u(x, t)$ is continuous in $(0, a] \times I$ on account of the arbitrariness of $\left(x_{0}, t_{0}\right)$. This proves $u \in C^{2}((0, a] \times I)$. The proof of Lemma 5.6 is complete.

\section{Appendix}

In this appendix let us prove Proposition 5.5. We consider the eigenvalue problem for $L_{\mu}, \mu>-1$,

$$
\left\{\begin{array}{l}
L_{\mu} \phi(x)=\lambda \phi(x), \quad x \in(0, a), \\
\phi(a)=0 .
\end{array}\right.
$$

Then it was shown in [Ya1] that (6.1) has the eigenvalues $\lambda_{j}$ and the corresponding eigenfunctions $\phi_{j}(x)$ which can be written by

$$
\lambda_{j}=\frac{\mu_{j}^{2}}{4(\mu+1) a}, \quad \phi_{j}(x)=\frac{1}{a^{1 / 2} J_{\mu+1}\left(\mu_{j}\right)} \frac{J_{\mu}\left(\mu_{j} \sqrt{x / a}\right)}{x^{\mu / 2}},
$$

where $\left\{\mu_{j}: j \in N\right\}$ is the set of all positive zero points of the $\mu$-order Bessel function $J_{\mu}(x)$ with $\mu_{1}<\mu_{2}<\ldots$. For several properties of the Bessel functions and their zero points, see [T] and [W]. It should be noted that $\left\{\phi_{j}\right\}$ is CONS in $L^{2}\left(0, a ; x^{\mu}\right)$ and a complete and orthogonal system in $H_{0}^{1}\left(0, a ; x^{\mu}\right)$.

Now let us expand $f \in L^{2}\left(0, a ; x^{\mu}\right)$ into the Bessel-Fourier series

$$
f=\sum_{j} f_{j} \phi_{j}, \quad f_{j}=\left(f, \phi_{j}\right)_{L^{2}\left(0, a ; x^{\mu}\right)} .
$$

Let us introduce a subspace $W^{\alpha}(0, a)(\alpha \geqq 0)$ of $L^{2}\left(0, a ; x^{\mu}\right)$ whose element $f$ satisfies

$$
\sum_{j} \lambda_{j}^{\alpha} f_{j}^{2}<+\infty
$$

Then $W^{\alpha}(0, a)$ is a Hilbert space with inner product

$$
(f, g)_{W^{\alpha}(0, a)}=\sum_{j} \lambda_{j}^{\alpha} f_{j} g_{j},
$$

where $f=\sum f_{j} \phi_{j}$ and $g=\sum g_{j} \phi_{j}$. The crucial point of $W^{s}(0, a)$ is given by the following proposition.

Proposition 6.1 ([Ya1]). Let $s \in Z_{+}$and $\mu>-1$. Then $W^{s}(0, a)$ is isomorphic to $K^{s}\left(0, a ; x^{\mu}\right)$, i.e.,

$$
C_{0}|f|_{H^{s}\left(0, a ; x^{\mu}\right)} \leqq|f|_{W^{s}(0, a)} \leqq C_{1}|f|_{H^{s}\left(0, a ; x^{\mu}\right)},
$$

holds for $f \in K^{s}\left(0, a ; x^{\mu}\right)$, where $C_{0}, C_{1}>0$ depend on $s, \mu$. 
We can also define the fractional power of $L_{\mu}$ by

$$
L_{\mu}^{\alpha} f=\sum_{j} \lambda_{j}^{\alpha} f_{j} \phi_{j}
$$

for all $\alpha \geq 0$. Then it was shown in [Ya1] that there exists a constant $C>1$ such that

$$
C^{-1}|f|_{W^{2 \alpha}} \leq\left|L^{\alpha} f\right|_{L^{2}} \leq C|f|_{W^{2 \alpha}}
$$

for all $f \in W^{\alpha}(0, a)$.

Now we consider BVP

$$
L_{\mu} w=g, \quad w(a)=0 .
$$

At first we prove the unique solvability to $(\mathrm{EP})$ in $K^{s}\left(0, a ; x^{\mu}\right)$.

LEMMA 6.2. If $g \in K^{s}\left(0, a ; x^{\mu}\right)$ for $s \in Z_{+}$, then (EP) has a unique solution $w$ in $K^{s+2}\left(0, a ; x^{\mu}\right)$ satisfying $(5.8)$.

PROOF. Substituting $g=\sum_{j} g_{j} \psi_{j}$ and $w=\sum_{j} w_{j} \psi_{j}$ into (EP) and comparing each of the Fourier coefficients, we have $w_{j}=g_{j} / \lambda_{j}$. Hence we obtain

$$
\sum_{j} \lambda_{j}^{s+2}\left|w_{j}\right|^{2} \leqq \sum_{j} \lambda_{j}^{s}\left|g_{j}\right|^{2}
$$

By using Proposition 6.1, $w$ belongs to $K^{s+2}\left(0, a ; x^{\mu}\right)$ and (5.8) holds. The uniqueness is clear from (5.8).

ProOf OF Proposition 5.5. We prove Proposition 5.5 by the induction argument. The case $s=1$ is clear from Lemma 6.2. Assuming that the statement of Proposition 5.5 holds for $s-1(s \geqq 2$ ), we will prove the statement of Proposition 5.5 for $s$ by establishing the regularity of solutions inside of $(0, a)$ and near $x=a$. Let us introduce a function space $B$ of all functions $\alpha(x)$ of $C^{\infty}$ in $[0, a]$ such that

$$
\alpha(x)= \begin{cases}1, & x \in[0, \xi], \\ 0, & x \in[\eta, a],\end{cases}
$$

for some $\xi, \eta$ with $0<\xi<\eta<a$.

As the first step we check the regularity of solutions in the interior of $(0, a)$. More precisely, we will see $\alpha w \in K^{s+2}\left(0, a ; x^{\mu}\right)$ for any $\alpha \in B$ by using so called bootstrap method. It follows from the equation in (EP) that

$$
L_{\mu}(\alpha w)=-\alpha g-\frac{2 \partial_{x} \alpha}{\mu+1}\left(x \partial_{x} w\right)-\left(L_{\mu} \alpha\right) w .
$$

Since $w$ belongs to $H^{s+1}\left(0, a ; x^{\mu}\right)$ by the assumption on induction, both $x \partial_{x} w$ and $w$ belong to $H^{s}\left(0, a ; x^{\mu}\right)$. In fact, it is enough to show that

$$
\int_{0}^{a} x^{\mu+j}\left\{\partial_{x}^{j}\left(x \partial_{x} w\right)\right\}^{2} d x<+\infty, \quad j=0,1, \ldots, s .
$$


It follows from the Leibniz formula that

$$
\partial_{x}^{j}\left(x \partial_{x} w\right)=x \partial_{x}^{j+1} w+j \partial_{x}^{j} w
$$

and hence, we have

$$
\begin{gathered}
\int_{0}^{a} x^{\mu+j}\left(x \partial_{x}^{j+1} w\right)^{2} d x=\int_{0}^{a} x^{\mu+j+2}\left(\partial_{x}^{j+1} w\right)^{2} d x<+\infty \\
\int_{0}^{a} x^{\mu+j}\left(j \partial_{x}^{j} w\right)^{2} d x<+\infty
\end{gathered}
$$

which proves (6.3). Clearly if $\alpha \in B$ and $f \in H^{s}\left(0, a ; x^{\mu}\right)$, then $\alpha f$ belongs to $K^{s}\left(0, a ; x^{\mu}\right)$. Hence $\beta x \partial_{x} w \in K^{s}\left(0, a ; x^{\mu}\right)$ for any $\beta \in B$, which implies that the right-hand side of (6.2) belongs to $K^{s}\left(0, a ; x^{\mu}\right)$. Therefore, using Lemma 6.2 , we get $\alpha w \in K^{s+2}\left(0, a ; x^{\mu}\right)$ for any $\alpha \in B$.

As the second step, we check that (5.8) holds for $w$ replaced by $\alpha w$. Applying (5.8) in Lemma 6.2 to (6.2), we have

$$
\begin{aligned}
&|\alpha w|_{H^{s+2}\left(0, a ; x^{\mu}\right)} \leqq C\left|-\alpha g-\frac{2 \partial_{x} \alpha}{\mu+1}\left(x \partial_{x} w\right)-\left(\frac{x}{\mu+1} \partial_{x}^{2} \alpha+\partial_{x} \alpha\right) w\right|_{H^{s}\left(0, a ; x^{\mu}\right)} \\
& \leqq C\left(|g|_{H^{s}\left(0, a ; x^{\mu}\right)}+\left|x \partial_{x} w\right|_{H^{s}\left(0, a ; x^{\mu}\right)}+|w|_{H^{s}\left(0, a ; x^{\mu}\right)}\right) \\
& \leqq C\left(|g|_{H^{s}\left(0, a ; x^{\mu}\right)}+|w|_{H^{s+1}\left(0, a ; x^{\mu}\right)}\right) \\
& \leqq C\left(|g|_{H^{s}\left(0, a ; x^{\mu}\right)}+|g|_{H^{s-1}\left(0, a ; x^{\mu}\right)}\right) \\
& \leqq C|g|_{H^{s}\left(0, a ; x^{\mu}\right)}
\end{aligned}
$$

with a certain constant $C$ depending on $\alpha$ and $m$, where we have used the assumption on induction.

As the third step, we check the regularity of $w$ at the boundary $x=a$ and prove the estimate (5.8). Given a constant $\delta \in(0, a)$, let us define a set $E_{\delta}$ of all $\gamma \in C^{\infty}((0, a])$ such that

$$
\gamma(x)= \begin{cases}1, & x \in[a-\delta / 2, a], \\ 0, & x \in[0, a-\delta] .\end{cases}
$$

Then (6.2) holds even for $\alpha$ replaced by $\gamma$ in the interval $(a-\delta, a)$. In this case $L_{\mu}$ is not degenerate and is a strongly elliptic operator in $(a-\delta, a)$. Hence the regularity theorem on strongly elliptic operator (Nirenberg theorem) tells us that $\gamma w$ belongs to $H^{s+2}\left(0, a ; x^{\mu}\right)$ for any $\gamma \in E_{\delta}$. In order to obtain the estimate of $|\gamma w|_{H^{s+2}\left(0, a ; x^{\mu}\right)}$, we use the representation of the solutions of equation in (EP):

$$
w(x)=-\int_{a}^{x} \frac{\mu+1}{s^{\mu+1}}\left(\int_{a}^{s} y^{\mu} g(y) d y\right) d s .
$$


Using this representation formula, we can write the derivatives of $w(x)$ by

$$
w^{(j)}(x)=\sum_{k=0}^{j-2} Q_{k}(x) g^{(k)}(x)+Q_{j}(x)\left(\int_{a}^{x} y^{\mu} g(y) d y\right)
$$

for $j=1, \ldots, s+2$, where $Q_{k}(x)$ are the polynomials of $1 / x$. Up to now, it is sufficient to estimate the top term $\left|x^{\frac{s+2}{2}}(\gamma w)^{(s+2)}\right|_{L^{2}\left(0, a ; x^{\mu}\right)}$, since the lower derivatives can be treated more easily. Since

$$
\left|x^{\frac{s+2}{2}}(\gamma w)^{(s+2)}\right|_{L^{2}\left(0, a ; x^{\mu}\right)}^{2}=\int_{a-\delta}^{a} x^{\mu+s+2}\left|(\gamma w)^{(s+2)}\right|^{2} d x \leqq C \int_{a-\delta}^{a} x^{\mu+s+2} \sum_{j=0}^{s+2}\left|w^{(j)}\right|^{2} d x,
$$

it follows from (6.5) that

$$
\left|x^{\frac{s+2}{2}}(\gamma w)^{(s+2)}\right|_{L^{2}\left(0, a ; x^{\mu}\right)}^{2} \leqq C \int_{a-\delta}^{a} x^{\mu+s+2} \sum_{j=0}^{s}\left|g^{(j)}\right|^{2} d x \leqq C|g|_{H^{s}\left(0, a ; x^{\mu}\right)}^{2} .
$$

Thus we get the required estimate

$$
|\gamma w|_{H^{s+2}\left(0, a ; x^{\mu}\right)} \leqq C|g|_{H^{s}\left(0, a ; x^{\mu}\right)} .
$$

Writing $w=\alpha w+(1-\alpha) w, \alpha \in B, 1-\alpha \in E_{\delta}$ for some $\delta \in(0, a)$, we conclude that $w$ belongs to $H^{s+2}\left(0, a ; x^{\mu}\right)$. Moreover, we obtain (5.8). In fact, we see from (6.4) and (6.6) that

$$
|w|_{H^{s+2}\left(0, a ; x^{\mu}\right)} \leqq|\alpha w|_{H^{s+2}\left(0, a ; x^{\mu}\right)}+|(1-\alpha) w|_{H^{s+2}\left(0, a ; x^{\mu}\right)} \leqq C|g|_{H^{s}\left(0, a ; x^{\mu}\right)} .
$$

This completes the proof of Proposition 5.5.

Acknowledgements. We would like to express our deep gratitude to Professor T. Matsuyama for his valuable comments, reading in detail and correcting the manuscript. We would like to also thank Mr. Y. Kosugi for providing us several techniques and calculations developed in his Master thesis of Tokai University.

\section{References}

[A] R. A. Adams, Sobolev Spaces, Academic Press, 1975.

[Ko] B. G. Korenev, Bessel Functions and their Applications, Taylor and Francis Inc., 2002.

[K-G-S] N. S. Koshlyakov, E. V. Gliner and M. M. Smirnov, Differential Equations of Mathematical Physics, Moscow, 1962 (in Russian). English Translation: North-Holland Publ. Co, 1964.

[Sath] J. SATHER, The existence of a global classical solution of the initial-boundary value problem for $\square u+u^{3}=f$, Arch. Rational Mech. Anal. 22 (1966), 292-307.

[T] C. J. Tranter, Bessel Functions with Some Physical Applications, Hart Publishing Co., New York, 1969.

[W] G. N. WATSON, Theory of Bessel Functions, Cambridge University Press, 1962.

[Wo-Ya] J. WongSAWASDI and M. YAMAGUCHI, Global solutions of IBVP to nonlinear equation of suspended string, Tokyo J. Math. 30, No. 2 (2007), 543-556. 
[Ya1] M. YAmAGUCHI, Almost periodic oscillations of suspended string under quasiperiodic linear force, J. Math. Anal. Appl. 303, No. 2 (2005), 643-660.

[Ya2] M. YAMAGUCHI, Free vibrations of nonlinear equation of suspended string, preprint.

[Ya-Na-Ma] M. Yamaguchi, T. NAGAi and K. Matsukane, Forced oscillations of nonlinear damped equation of suspended string, J. Math. Anal. Appl. 342, No. 2 (2008), 89-107.

Present Addresses:

JAIPONG WONGSAWASDI

Department of Mathematics, Faculty of Science,

King MongKut's Institute of TECHNOLOGY LADKRABANG,

CHALONGKRUNG LADKRABANG, BANGKOK 10520, THAILAND.

e-mail: kwjaipon@kmitl.ac.th

Masaru Yamaguchi

Department of Mathematics, Faculty of Science,

TOKAI UNIVERSITY,

HIRATSUKA, KANAGAWA 259-1292 JAPAN.

e-mail: yamagchi@sm.u-tokai.ac.jp 